\title{
CARACTERIZAÇÃO FÍSICO-QUÍMICA E SENSORIAL EM QUEIJO DE KEFIR COM E SEM CONDIMENTOS
}

\section{Physical-chemical and sensory characterization in kefir cheese with and without condiments}

\author{
Isabela Zottmann da Silval, Simone Weschenfelder ${ }^{I^{*}}$
}

\begin{abstract}
RESUMO
Queijos de kefir com e sem condimentos foram produzidos e analisados quanto às suas características físico-químicas e sensoriais. Foram produzidos dois tipos de queijo, sendo um tradicional e outro com condimentos como salsa, cebolinha, alho desidratado e pimenta preta. Análise de $\mathrm{pH}$ e determinação de umidade, cinzas, lipídios e lactose foram executadas nos queijos, bem como avaliação sensorial utilizando escala hedônica de 9 pontos para os atributos cor, consistência, aparência e sabor, cálculo do índice de aceitabilidade e a intenção de compra. Foram encontrados teores de umidade de 76,27\%, cinzas 1,16\%, lipídios $11,66 \%$, lactose de 2,33\% e pH de 3,86 no queijo sem condimentos, já no queijo com condimentos foram encontrados percentuais semelhantes de umidade $76,58 \%$, cinzas $2,23 \%$, lipídios $10 \%$, lactose de $2,05 \%$ e pH de 3,57. Na análise de sensorial encontrou-se índice de aceitabilidade maior que $70 \%$ em todos os parâmetros avaliados. Não houve diferença significativa entre os queijos para os parâmetros avaliados. Dessa forma, foi possível desenvolver duas formulações de queijo de kefir, uma com e outra sem condimentos. Ambas as formulações não apresentaram diferença significativa para os parâmetros físicoquímicos e sensoriais avaliados. Os resultados encontrados geram oportunidade para explorar cada vez mais os derivados de kefir.
\end{abstract}

Palavras-chave: escala hedônica; análise de variância; aceitabilidade; alimento funcional.

1 Universidade Feevale, Campus II, ERS-239, 2755, 93525-075, Novo Hamburgo, RS, Brasil. E-mail: simone.weschenfelder@yahoo.com.br

* Autor para correspondência 


\begin{abstract}
Kefir cheeses with and without condiments were produced and analyzed for their physical-chemical and sensory characteristics. Two types of cheese were produced, one traditional and the other with condiments such as parsley, chives, dehydrated garlic, and black pepper. $\mathrm{pH}$ analysis and determination of moisture, ash, lipids, and lactose were performed on the cheeses, as well as sensory evaluation using a 9-point hedonic scale for color, consistency, appearance, and flavor attributes, calculation of acceptability index, and purchase intention. Moisture contents of $76.27 \%$, ash $1.16 \%$, lipids $11.66 \%$, lactose $2.33 \%$, and $\mathrm{pH} 3.86$ were found in cheese without spices, whereas in the cheese with spices, similar contents of humidity $76.58 \%$, ash $2.23 \%$, lipids $10 \%$, lactose $2.05 \%$, and $\mathrm{pH} 3.57$ were found. In the sensory analysis, an acceptability index greater than $70 \%$ was found in all parameters evaluated. There was no significant difference between cheeses for the evaluated parameters. In this way, it was possible to develop two formulations of kefir cheese, one with and one without condiments. Both formulations showed no significant difference in the physicochemical and sensory parameters evaluated. The found results provide an opportunity to explore kefir derivatives even more.
\end{abstract}

Keywords: hedonic scale; variance analysis; acceptability; functional food.

\section{INTRODUÇ̃̃O}

O queijo é considerado um dos alimentos mais nutritivos que se conhece, que apresenta na sua constituição proteínas de alto valor biológico, carboidratos, lipídios e micronutrientes importantes como cálcio, fósforo, vitamina A e vitaminas do complexo B (PERRY, 2004).

O queijo é o produto fresco ou maturado, obtido pela separação parcial do soro do leite ou de soros lácteos, coagulados pela ação física do coalho, de enzimas específicas, de bactérias específicas, de ácidos orgânicos, isolados ou combinados. O queijo não pode conter na sua base láctea gordura e/ou proteínas que não sejam de origem animal. Pode conter ou não aditivos, outras substâncias alimentícias, condimentos, entre outros (BRASIL, 1996).

Condimentos e especiarias são definidos como produtos aromáticos de origem vegetal que são utilizados principalmente para conferir sabor aos alimentos. O uso destes produtos vem sendo cada vez mais explorado no mercado alimentício devido a procura por alimentos mais naturais e a preocupação em relação a saúde dos consumidores. Estes possuem propriedades benéficas à saúde do consumidor, como atividade antimicrobiana, antioxidante e medicinal (FURLAN, 2007; PEREIRA et al., 2006).

O uso de condimentos na preparação de alimentos pode ser uma alternativa para aumentar seu tempo de prateleira. Rodrigues et al. (2011) estudaram a atividade antibacteriana in vitro, predeterminada em 12 plantas (salsa, manjerona branca, manjerona preta, manjericão, sálvia, tomilho, anis verde, alfavaca, alho nirá, alho poró, cúrcuma e pimenta dedo-de-moça) utilizadas como condimentos, em uma preparação de frango cozido. A atividade antimicrobiana foi aferida a cada duas horas em 24 horas. Os 12 condimentos testados apresentaram atividade anti Escherichia coli, tornando-se alternativas para serem utilizadas de forma doméstica, garantindo um alimento seguro e sensorialmente aceito. 
Acredita-se que o início da produção de queijo foi dado a partir da domesticação de animais como vacas e cabras, onde acidentalmente o leite passava por um processo em que ocorria a separação de soro e massa, obtendo um produto com maior prazo de validade, qualidade sensorial e de fácil transporte. Estima-se que $30 \%$ da produção mundial de leite é reservada a fabricação de queijos, podendo existir mais de 1000 tipos de queijo através do mundo, além de incontáveis aplicações culinárias (FOX; McSWEENEY, 1998; FOX et al., 2000).

Dentre os diferentes tipos de microrganismos que podem ser empregados para fabricação do queijo, temos os que constituem os grãos de kefir. Os grãos de kefir apresentam a aparência semelhante à de uma couve-flor, com forma irregular e coloração amarelada ou esbranquiçada. Os grãos sãos massas gelatinosas que podem medir de 3 a $35 \mathrm{~mm}$ de diâmetro. A sua estrutura é composta por uma associação de leveduras, bactérias ácido-láticas, bactérias-acéticas, entre outros microrganismos, envolvidos por uma matriz de polissacarídeos chamados de kefiran (OTLES; CAGINDI, 2003; WESCHENFELDER, 2009; WESCHENFELDER et al., 2011).

De origem turca, a palavra kefir significa bem-estar, fenômeno que está ligado ao seu consumo. O kefir é um leite fermentado que pode ser produzido com facilidade em escala artesanal e os grãos de kefir são tradicionalmente adquiridos através de doações. Os métodos tradicionais de produção consistem na adição dos grãos de kefir em leite ou em uma mistura de água e açúcar mascavo (SANTOS et al., 2012; WSZOLEK et al., 2006).

O kefir é considerado um alimento funcional por conter probióticos, que são bactérias vivas associadas a efeitos benéficos em humanos e animais. Essas bactérias, se ingeridas de forma adequada, podem trazer benefícios a saúde do consumidor.
Considerando a produção em escala artesanal, o kefir se torna uma estratégia nutricional em comunidades de baixa renda, pois através dele é possível levar alimentos com propriedade funcional a população que não consegue adquirir produtos dessa linha em supermercados (ARAYA et al., 2006; GUARNER; SCHAAFSMA, 1998; VASILJEVIC; SHAH, 2008).

Os alimentos funcionais são aqueles que além de fornecerem a nutrição básica, possuem a capacidade de proporcionar saúde através de mecanismos que não são garantidos pela nutrição convencional. Estes são capazes de proporcionar a manutenção do bem-estar e saúde e/ou reduzir o risco de algumas doenças. Mas deve-se destacar que os alimentos funcionais são alimentos que promovem saúde e não a cura de doenças. Há um aumento na busca de alimentos funcionais nos mercados pelos consumidores que cada vez estão se preocupando mais com a sua saúde e bemestar, e se tornaram uma tendência para o mercado alimentício, que cada vez explora mais a elaboração destes produtos (HASLER, 2000; HEASMAN; MELLENTIN, 2001; ROBERFROID, 2002; SANDERS, 1998).

$\mathrm{Na}$ área da nutrição, o desenvolvimento de novos produtos que agreguem características diferenciadas é um desafio, pois além da questão nutricional o aspecto sensorial é muito importante. A análise sensorial é um dos métodos mais importantes para a avaliação de um novo produto, pois é o aspecto mais observado pelo consumidor. A decisão de compra é tomada dependendo de características sensoriais como odor, sabor, textura e aparência. Conhecer as características que são esperadas pelo consumidor se torna uma ferramenta indispensável para atingir as expectativas dos consumidores e se sobressair sobre seus concorrentes (DUTCOSKY, 2013; STONE; SIDEL, 1993). Assim, justifica-se a realização do presente estudo, que tem como objetivo produzir queijo de kefir com e sem 
condimentos e realizar sua avaliação sensorial e físico-química.

\section{MATERIAL E MÉTODOS}

Foram produzidas duas formulações de queijo de kefir, uma tradicional (A) e uma condimentada (B). Para a produção utilizou-se leite pasteurizado padronizado, homogeneizado e condimentos adquiridos no comércio local e os grãos de kefir oriundos de uma manipulação familiar artesanal. As formulações dos queijos A e B foram produzidas segundo Weschenfelder et al. (2011) adaptado, utilizando as Boas Práticas de Manipulação de Alimentos (BRASIL, 2004) ao longo do processamento.

A coagulação do leite foi feita a partir da fermentação com grãos de kefir. Para cada $1 \mathrm{~g}$ de grãos de kefir foram adicionados $10 \mathrm{~mL}$ leite pasteurizado padronizado e homogeneizado. Em seguida, o conteúdo passou por fermentação aeróbia em $25^{\circ} \mathrm{C}$ por 48 horas. Após este processo, realizou-se a filtração, utilizando uma peneira plástica esterilizada (12-mesh), separando o kefir e os grãos de kefir (que foram inoculados ao leite novamente). $\mathrm{O}$ produto obtido passou pelo processo de filtração, com a ajuda de um funil e filtro de café de papel (Melitta ${ }^{\circledR}$ ), por mais 24 horas, em geladeira $\left(5^{\circ} \mathrm{C}\right)$ para obtenção do queijo de kefir (WESCHENFELDER et al., 2011).

A condimentação foi feita com adição dos condimentos salsinha, cebolinha, alho desidratado e pimenta preta. A salsa e a cebolinha passaram pelo processo de pré-preparo e higienização, de acordo com a Portaria Estadual no 78/2009 (RIO GRANDE DO SUL, 2009). Para a obtenção do queijo condimentado (B), os ingredientes de origem vegetal utilizados foram (para cada $100 \mathrm{~g}$ de queijo): $5 \mathrm{~g}$ de salsa, $6 \mathrm{~g}$ de cebolinha, $1 \mathrm{~g}$ de alho desidratado e $0,5 \mathrm{~g}$ de pimenta preta. Estes ingredientes foram homogeneizados em um mixer e acrescidos ao queijo. As amostras de queijo (A e B) foram armazenadas em vidros com tampa, mantidas resfriadas na temperatura de $5^{\circ} \mathrm{C}$ para posteriormente realizar as análises físicoquímicas e sensoriais (WESCHENFELDER et al., 2011).

O processo de fabricação do queijo condimentado foi realizado por oito vezes até obter-se a padronização do produto, ou seja, até o queijo apresentar as mesmas características físico-químicas. Estas repetições se fazem necessárias, pois os grãos de kefir apresentam características que variam de acordo com a sua origem e manipulação e que tendem a padronização quando submetidas às mesmas variáveis ao longo do tempo (WESCHENFELDER et al., 2011). Todo o experimento foi realizado em três repetições e as análises físico-químicas de determinação de $\mathrm{pH}$, umidade, cinzas, lactose e lipídios dos queijos foram mensuradas em triplicata de acordo com os métodos propostos pelo Instituto Adolfo Lutz (ZENEBON et al., 2008).

A análise sensorial dos queijos e de intenção de compra foram realizadas um dia após a fabricação do produto, ambas aplicadas no mesmo momento. Foram convidados a participar da avaliação 28 alunos e funcionários de uma universidade, classificados como avaliadores sensoriais não treinados. Foram utilizados como critério de exclusão indivíduos que apresentassem intolerância à lactose ou alergia a algum dos ingredientes listados (DUTCOSKY, 2013).

Os testes foram aplicados em uma sala de aula adaptada. A avaliação foi realizada de forma individualizada, respeitando o critério de duas horas antes ou após almoço (DUTCOSKY, 2013; SOUZA, 2006).

Cada avaliador recebeu inicialmente uma amostra dos queijos A e B junto com biscoito de água e sal, que serviu de base, e um copo de água para ser ingerido após a avaliação, a 
fim de reduzir efeitos de fadiga e adaptação olfatória, acompanhado da ficha de análise sensorial e intenção de compra. Os avaliadores receberam uma ficha com escala hedônica de 9 pontos: sendo 1 "desgostei extremamente" e 9 "gostei extremamente", para a avaliação do produto quanto a cor, consistência, aparência e sabor (Figura 1). Junto com a avaliação sensorial, realizou-se uma pesquisa de intenção de compra, contendo as seguintes as opções: "compraria" ou "não compraria", para cada tipo de queijo (DUTCOSKY, 2013; WESCHENFELDER et al., 2011). O índice de aceitabilidade (IA) foi calculado através da equação IA $(\%)=\mathrm{A} \times 100 / \mathrm{B}$, onde: $\mathrm{A}=$ nota média obtida para o produto; $\mathrm{B}=$ nota máxima dada ao produto. Dutcosky (2013) defende que para o índice de aceitabilidade ser considerado satisfatório, deve apresentar o valor de igual ou maior de $70 \%$.

$\mathrm{O}$ presente projeto foi submetido à apreciação do Comitê de Ética e Pesquisa da Universidade Feevale, RS, sendo aprovado com o número 17637219.7.0000.5348.

\section{RESULTADOS E DISCUSSÃO}

A acidez característica dos produtos

\section{ESCALA HEDÔNICA DE AVALIAÇÃO E INTENÇÃO DE COMPRA Teste de aceitação em escala hedônica \\ Nome (optativo): Idade: anos \\ Sexo: Data:}

Você está recebendo duas diferentes amostras de queijo. Utilizando a escala abaixo, avalie as amostras para descrever o quanto você gostou ou desgostou dos produtos em relação a cor, consistência, aparência e sabor. Entre uma amostra e outra, beba um copo de água.

1 Desgostei extremamente

2 Desgostei muito

3 Desgostei moderadamente

4 Desgostei ligeiramente

5 Indiferente

6 Gostei ligeiramente

7 Gostei moderadamente

8 Gostei muito

9 Gostei extremamente

Você deverá avaliar os critérios abaixo, e atribuir uma das notas conforme as opções acima.

\begin{tabular}{|l|l|l|l|l|}
\hline Formulação & Cor & Consistência & Aparência & Sabor \\
\hline & & & & \\
\hline & & & & \\
\hline
\end{tabular}

Caso estas formulações estivessem disponíveis para venda, você compraria?

( ) Sim. Qual?

( ) Não.

Figura 1 - Ficha de resposta utilizada na avaliação sensorial dos queijos 
derivados da fermentação de grãos de kefir se dá pela degradação da lactose pelos microrganismos, formando o ácido lático (SILVA JÚNIOR, 1995). A diminuição do $\mathrm{pH}$ acontece sempre que a lactose é degradada. No estudo Weschenfelder et al. (2011), foram encontrados valores de 3,59 a 3,79 no $\mathrm{pH}$ nos derivados de kefir com o tempo de fermentação 168 horas. Em outro estudo realizado por Weschenfelder et al. (2018a) os valores de pH encontrados em queijo de kefir foram de 4,8 e 5,0 em 48 horas de fermentação. Já no queijo de kefir do presente estudo o $\mathrm{pH}$ manifestou valores semelhantes, de 3,86 e 3,57, na formulação A e na formulação B, respectivamente. Os resultados podem variar muito, pois a composição microbiológica dos grãos de kefir são extremamente variáveis dependendo das condições de armazenamento, manipulação, matéria prima, tempo e técnicas utilizadas no preparo (MICHELI et al., 1999).

As avaliações da composição química das duas formulações de queijo estão apresentadas na Tabela 1 e sinalizam que não houve diferença significativa entre as formulações de queijo para os parâmetros avaliados.

No presente estudo foram encontrados os percentuais de lactose de 2,33\% e 2,05\% nas formulações A e B de queijo de kefir, respectivamente. No estudo de Weschenfelder et al. (2011), os teores de lactose ficaram entre $0,93 \%$ na formulação A e 1,59\% na formulação B (ambos fermentaram por 168 horas) em formulações de kefir. As formulações também possuíam diferentes concentrações de leite e grãos de kefir, sendo a A $1: 10$ e a B $1: 15$.

No estudo de Irigoyen et al. (2005), que avaliou as características microbiológicas, físico-químicas e sensoriais de kefir sob armazenamento de até 28 dias, o teor de lactose apenas se alterou nas primeiras 24 horas de fermentação e apresentou diminuição de $20 \%$ a $25 \%$ do teor de lactose presente no leite, após este período os valores permaneceram constantes.

Os queijos A e B apresentaram percentuais de cinzas de $1,16 \%$ e $2,23 \%$, valores semelhantes ao encontrado por Weschenfelder et al. (2018a) em duas formulações de queijo de kefir, que foram de $0,59 \%$ e $1,32 \%$. Estes teores são os minerais como cálcio, potássio, magnésio, sódio e zinco oriundos do próprio leite. Isto se torna positivo, considerando que estes micronutrientes devem estar presentes na alimentação diária, favorecendo a saúde da população, que costuma apresentar ingestões inadequadas de micronutrientes ao longo da vida (CEMBRANEL et al., 2012; PINHEIRO et al., 2018).

Tabela 1 - Valores médios e desvios padrão atribuídos aos parâmetros lactose, cinzas, umidade e lipídios de queijo de kefir tradicional e com condimentos

\begin{tabular}{ccccc}
\hline Queijos de kefir & Lactose (\%) & Cinzas (\%) & Umidade (\%) & Lipídios (\%) \\
\hline $\begin{array}{c}\text { Queijo de kefir } \\
\text { tradicional (A) }\end{array}$ & $2,33( \pm 0,21)^{\mathrm{a}}$ & $1,16( \pm 0,14)^{\mathrm{a}}$ & $76,27( \pm 0,47)^{\mathrm{a}}$ & $11,66( \pm 1,52)^{\mathrm{a}}$ \\
$\begin{array}{c}\text { Queijo de kefir com } \\
\text { condimentos (B) }\end{array}$ & $2,05( \pm 0,03)^{\mathrm{a}}$ & $2,23( \pm 0,93)^{\mathrm{a}}$ & $76,58( \pm 0,17)^{\mathrm{a}}$ & $10,0( \pm 1,0)^{\mathrm{a}}$ \\
\hline
\end{tabular}

a Médias seguidas por letras iguais na mesma coluna não diferem significativamente entre si pelo Teste de Tukey a um nível de confiança de $95 \%$. 
Os queijos de kefir A e B estudados apresentaram percentuais de umidade de $76,27 \%$ e $76,58 \%$, respectivamente. Semelhante ao queijo de kefir elaborado por Weschenfelder et al. (2011) que encontraram percentual de $79,39 \%$ e $79,41 \%$ em duas formulações de leban (queijo).

O percentual de umidade está diretamente relacionado ao valor calórico dos queijos, quanto menor a umidade, maior é o percentual de lipídeos e proteínas do queijo. Segundo a legislação vigente (BRASIL, 1996), o queijo com umidade superior a $55 \%$ é considerado um queijo de muita alta umidade. Isso classifica o queijo de kefir (A e B) como um alimento perecível, reforçando que apesar da atividade antimicrobiana apresentada por diversos autores pela fermentação de kefir (WESCHENFELDER et al., 2009; WESCHENFELDER et al., 2018a; WESCHENFELDER et al., 2018b), deve-se ter cuidado com a manipulação, armazenamento e matérias prima utilizadas.

Os queijos de kefir A e B apresentaram teores de lipídios de $11,66 \%$ e $10 \%$, o que correspondeu $49,13 \%$ e $42,70 \%$ de gordura no extrato seco (GES) respectivamente. Dessa forma, o queijo A pode ser classificado pela legislação vigente (BRASIL, 1996) como gordo, por conter percentuais entre 45,0 e $59,9 \%$ de GES, enquanto o queijo B classificase como semigordo (entre 25,0 e 44,9\% de GES). Sperotto et al. (2017), ao avaliar o percentual de lipídios em queijo de Kefir, encontrou percentual de $18,16 \%$.

Mesmo não sendo determinado em laboratório, analisando os dados apresentados na Tabela 1 podemos estimar o teor de proteína do queijo A em aproximadamente $8,58 \%$, semelhante à formulação $\mathrm{B}$, de $9,14 \%$. Valores próximos ao encontrado por Sperotto et al. (2017), que analisaram as características físico-químicas de um queijo cremoso de kefir, com $11,79 \%$ de proteína. As proteínas encontradas no queijo de kefir são designadas como proteínas de alto valor biológico, pois são derivadas de matéria prima animal, assim, possuem em sua composição todos os aminoácidos essenciais (PIRES et al., 2006).

Moreira (2019), ao definir as informações necessárias para elaborar o rótulo de queijo de kefir, encontrou percentual de proteína de $12,81 \%$, gorduras totais $7,8 \%$, umidade $64,01 \%$, cinzas $2,37 \%$, corroborando com o presente estudo.

Para determinar a qualidade de um produto existem três aspectos cruciais, são eles físico-químicos (nutricionais), sensoriais e microbiológicos. O aspecto sensorial é o mais ligado a escolha do produto pelo consumidor final (DUTCOSKY, 2013).

A análise sensorial contou com a participação de 28 voluntários, sendo 4 do sexo masculino e 24 do sexo feminino. Estes avaliaram os queijos de acordo com cor, consistência, aparência e sabor, conforme pode ser observado na Tabela 2 .

O queijo temperado (B) obteve os seguintes percentuais de aceitação, quando calculado o índice de aceitabilidade: $83 \%$ para o item cor, $85 \%$ para consistência, $84 \%$ para a aparência e $81 \%$ para o sabor. O queijo tradicional (A) obteve resultados semelhantes: $80 \%$ para cor, $80 \%$ para consistência, $81 \%$ para aparência e $71 \%$ para sabor. O índice de aceitabilidade (IA) nas formulações A e $\mathrm{B}$, em relação a cor, consistência, aparência e sabor foi superior a $70 \%$, ou seja, segundo Ducosky (2013), os queijos (A e B) possuem significante aceitação e teriam impacto positivo se lançados no mercado. Já avaliação de intenção de compra, entre os 28 avaliadores, 15 comprariam o queijo com condimentos, 7 o queijo tradicional, 4 comprariam ambas as formulações e apenas 2 não comprariam nenhum dos queijos apresentados.

Em um estudo realizado por Weschenfelder et al. (2011) foram produzidas 3 formulações de antepasto a base de kefir leban (queijo). A primeira continha salsa, 
cebola, alho nirá, pimenta preta, sal e leban; a segunda preparação com tomate, pimentão, açúcar e leban; e a terceira era feita de hibisco, açúcar e leban. Na análise sensorial e intenção de compra das formulações, a com maior aceitação foi a primeira citada. Este resultado pôde ser justificado pelo uso de condimentos que são tradicionalmente conhecidos e estão presentes nas preparações culinárias dos brasileiros, e ainda pode ter mascarado a tradicional acidez presente nos produtos originados da fermentação de grãos de kefir. Resultados semelhantes ao presente estudo, que teve maior percentual de aceitação e intenção de compra na formulação com adição de condimentos (B) para todos os parâmetros avaliados.

Em um estudo realizado em 2019 (PLETSCH et al., 2019), foi criado um gelado comestível utilizando kefir, polpa de jabuticaba e morango para posterior análise sensorial. Observou-se através da análise sensorial que os quatro requisitos avaliados, textura, odor, sabor e cor, apresentaram boa aceitabilidade, superior a $80 \%$ durante todo o período avaliado. Em outro estudo, realizado por Santos et al. (2012), foram produzidos quatro tipos de queijo tipo Petit Suisse, duas formulações salgadas e duas formulações doces. Foi realizada a análise sensorial, avaliando aceitação geral e sabor.
A formulação salgada preparada com ervas recebeu nota 7 nas duas análises. Já Sperotto et al. (2017), ao analisarem uma formulação de queijo de kefir, obtiveram índice de aceitabilidade superior a $88 \%$ nos requisitos sabor, textura, aroma e aparência geral. Produtos obtidos através da fermentação de kefir possuem bom percentual de aceitação (PLETSCH et al., 2019).

O kefir é um alimento funcional que contém microrganismos probióticos; (SALMINEN et al., 1998; SANTOS et al., 2012), sendo assim é possível sugerir que os queijos produzidos através de kefir podem apresentar potencial probiótico. Ainda é necessário a realização de mais estudos para afirmar a alegação de propriedade funcional, sendo uma alternativa a ser explorada no nicho de mercado que está em desenvolvimento no Brasil e que já movimenta cerca de milhões de dólares ao ano nos Estados Unidos (HEASMAN; MELLENTIN, 2001; VIEIRA et al., 2006).

\section{CONCLUSÃO}

Foi possível desenvolver e avaliar a qualidade de duas formulações de queijo de kefir, uma com e outra sem condimentos. Ambas as formulações não apresentaram diferença significativa para os parâmetros

Tabela 2 - Valores médios e desvios padrão atribuídos às variáveis cor, consistência, aparência e sabor de queijo de kefir tradicional e com condimentos

\begin{tabular}{ccccc}
\hline Queijos de kefir & Cor & Consistência & Aparência & Sabor \\
\hline $\begin{array}{c}\text { Queijo de kefir } \\
\text { tradicional (A) }\end{array}$ & $7,21( \pm 1,45)^{\mathrm{a}}$ & $7,21( \pm 1,64)^{\mathrm{a}}$ & $7,32( \pm 1,36)^{\mathrm{a}}$ & $6,43( \pm 1,91)^{\mathrm{a}}$ \\
$\begin{array}{c}\text { Queijo de kefir com } \\
\text { condimentos (B) }\end{array}$ & $7,54( \pm 1,4)^{\mathrm{a}}$ & $7,64( \pm 0,99)^{\mathrm{a}}$ & $7,57( \pm 1,23)^{\mathrm{a}}$ & $7,32( \pm 1,81)^{\mathrm{a}}$ \\
\hline
\end{tabular}

a Médias seguidas por letras iguais na mesma coluna não diferem significativamente entre si pelo Teste de Tukey a um nível de confiança de $95 \%$. 
físico-químicos e sensoriais avaliados. O estudo demonstrou que o queijo de kefir obteve uma boa aceitação entre os 28 avaliadores, com índices de aceitabilidade superiores a $70 \%$.

Os resultados encontrados propõem oportunidade para explorar cada vez mais os derivados de kefir, que se apresentam como produtos inovadores e diferenciados no mercado, destacando o papel fundamental do nutricionista no desenvolvimento de produtos na indústria de alimentos.

\section{REFERÊNCIAS}

ARAYA, M. et al. Probiotics in food: Health and nutritional properties and guidelines for evaluation. Report of a joint FAO/WHO expert consultation on evaluation of health and nutritional properties of probiotics in food including powder milk with live lactic acid bacteria. FAO Food and Nutrition paper. 85. Rome: FAO/WHO, 2006. 56 p. Disponível em: http://www.fao.org/3/a-a0512e.pdf. Acesso em: 25 fev. 2019.

BRASIL. Ministério da Agricultura, do Abastecimento e da Reforma Agrária. Portaria $\mathrm{n}^{\circ}$ 146, de 7 de março de 1996. Aprova os Regulamentos Técnicos de Identidade e Qualidade dos Produtos Lácteos. Diário Oficial da República Federativa do Brasil: seção 1, Brasília, DF, n. 48, p. 3977, 11 mar. 1996.

BRASIL. Ministério da Saúde. Agência Nacional de Vigilância Sanitária. Resolução RDC no 216, de 15 de setembro de 2004. Dispõe sobre Regulamento Técnico de Boas Práticas para Serviços de Alimentação. Diário Oficial da União: seção 1, Brasília, DF, n. 179 , p. 25,16 set 2004.

CEMBRANEL, F.; GONZALEZ-CHICA, D. A.; D'ORSI, E. Inadequações na ingestão dietética de micronutrientes por homens e mulheres residentes no Sul do Brasil: Estudo EpiFloripa Adultos 2012. Cadernos de Saúde Pública, v. 32, n. 8, e00164015, 2016. DOI: 10.1590/0102-311X00164015.

DUTCOSKY, S. D. Análise Sensorial de Alimentos. 4. ed. ampl. e rev. Curitiba: PUCPRess, 2013.

FOX, P. F. et al. Fundamentals of Cheese Science. 1. ed. Gaithersburg: Aspen Publishers, 2000. 544 p.

FOX, P. F.; McSWEENEY, P. L. H. Dairy Chemistry and Biochemistry. 1. ed. London: Blackie Academic \& Professional, 1998. $478 \mathrm{p}$.

FURLAN, M. R. Dossiê Técnico: Cultivo de plantas condimentares herbáceas. Belo Horizonte: Fundação Centro Tecnológico de Minas Gerais, 2007. 29 p. Disponível em: http://www.respostatecnica.org.br/dossietecnico/downloadsDT/MTk1. Acesso em: 02 maio 2019.

GUARNER, F.; SCHAAFSMA, G. J. Probiotics. International Journal of Food Microbiology, v. 39, n. 3, p. 237-238, 1998. DOI: 10.1016/S0168-1605(97)00136-0.

HASLER, C. M. The changing face of functional foods. Journal of the American College of Nutrition, v. 19, n. 5, p. 499S-506S, 2000. DOI: $10.1080 / 07315724.2000 .10718972$.

HeAsman, M.; MELlentin, J. The Functional Foods Revolution: Healthy people, healthy profits? London: Earthscan, 2001.

IRIGOYEN, A. et al. Microbiological, physicochemical, and sensory characteristics of kefir during storage. Food Chemistry, 
v. 90, p. 613-620, 2005. DOI: 10.1016/j. foodchem.2004.04.021

MICHELI, L. et al. Isolation and characteriSation of a ropy lactobacillus strain producing the exopolysaccharide Kefiran. Applied Microbiology and Biotechnology, v. 53 , n. 1, p. 69-74, 1999. DOI: $10.1007 /$ s002530051616.

\section{MOREIRA, T. A. Adequação da rotulagem} de queijo de kefir. 2019. 33 f. Trabalho de Conclusão de Curso (Engenharia de Alimentos) - Universidade Tecnológica Federal do Paraná, Campo Mourão, 2019.

OTLES, S.; CANGINDI, O. Kefir: a probiotic dairy-composition, nutritional and therapeutic aspects. Pakistan Journal of Nutrition, v. 2, n. 2, p. 54-59, 2003.

PEREIRA, M. C. et al. Inibição do desenvolvimento fúngico através da utilização de óleos essenciais de condimentos. Ciência e Agrotecnologia, v. 30, n. 4, p. 731-738, 2006. DOI: $10.1590 / \mathrm{S} 1413-70542006000400020$.

PERRY, S. P. K., Queijos: aspectos químicos, bioquímicos e microbiológicos. Química Nova, v. 27, n. 2, p. 293-300, 2004.

PINHEIRO, L. F. et al. Inadequação da ingestão de micronutrientes de acordo com as DRIs (Dietary Reference Intakes) em Adolescentes. International Journal of Nutrology, v. 11, n. 1, 2018. DOI: 10.1055/s0038-1674758.

PIRES, C. V. et al. Qualidade nutricional e escore químico de aminoácidos de diferentes fontes proteicas. Ciência e Tecnologia de Alimentos, v. 26, n. 1, p. 179-187, 2006. DOI: 10.1590/S0101-20612006000100029.

PLETSCH, L. B. H. et al. Gelado comestível de kefir adicionado de polpa de jabuticaba e morango. Revista do Instituto de Laticínios Cândido Tostes, v. 74, n. 1, p. 223-234, 2019. DOI: 10.14295/2238-6416.v74i1.701.

RIO GRANDE DO SUL. Secretaria da Saúde. Portaria Estadual $n^{\circ} 78$ de 28 de janeiro de 2009. Aprova a lista de verificação em boas práticas para serviços de alimentação, aprova normas para cursos de capacitação em boas práticas para serviços de alimentação e dá outras providências. Diário Oficial do Estado do Rio Grande do Sul: edição 1, Porto Alegre, RS, p. 35, 30 jan. 2009.

ROBERFROID, M. B. Functional food concept and its application to prebiotics. Digestive and Liver Disease, v. 34, n. 2, p. S105-S110, 2002. DOI: 10.1016/S15908658(02)80176-1.

RODRIGUES, F.; CARVALHO, H. H. C.; WIEST, J. M. Diferentes condimentos vegetais: Avaliação sensorial e de atividade antibacteriana em preparação alimentar com frango cozido. Revista Brasileira de Plantas Medicinais, v. 13, n. 3, p. 342-348, 2011. DOI: $10.1590 / \mathrm{S} 1516-05722011000300$ 10.1590/S1516-05722011000300014.

SALMINEN, S. et al. Functional food science and gastrointestinal physiology and function. British Journal of Nutrition, v. 80, supl. 1, p. S147-S171, 1998. DOI: 10.1079/ bjn19980108.

SANDERS, M. E. Overview of functional foods: Emphasis on probiotic bacteria. International Dairy Journal, v. 8, n. 5-6, p. 341-347, 1998. DOI: 10.1016/S09586946(98)00056-9.

SANTOS, F. L. et al. "Petit suisse" cheese from kefir: an alternative dessert with microorganisms of probiotic activity. 
Ciência e Tecnologia de Alimentos, v. 32, n. 3, p. 485-491, 2012. DOI: 10.1590/S010120612012005000077.

SILVA JÚNIOR, E. A. Manual de Controle Higiênico Sanitário em Alimentos. São Paulo: Varela, 1995.

SOUZA, A. Elaboração e análise sensorial de um coquetel laxante à base de kefir e frutas em pacientes do Hospital Universitário Onofre Lopes. 2006. Trabalho de Conclusão de Curso (Graduação) - Universidade Federal do Rio Grande do Norte, Natal, 2006.

SPEROTTO, L. et al. Desenvolvimento de queijo cremoso com kefir: Análises sensoriais e físico-químicas. Revista Eletrônica Biociências, Biotecnologias e Saúde, n. 18, p. 60-68, 2017.

STONE, H.; SIDEL, J. Sensory Evaluation Statistics Practices. New York: Elsevier, 1993.

VASILJEVIC, T.; SHAH, N. P. Probiotics From Metchnikoff to bioactives. International Dairy Journal, v. 18, n. 7, p. 714-728., 2008. DOI: 10.1016/j.idairyj.2008.03.004.

VIEIRA, A. C. P.; CORNÉlio, A. R.; SALGADO, J. M. Alimentos funcionais: Aspectos relevantes para o consumidor. Jus Navigandi, n. 1123, 2006. Disponível em: http://jus2.uol.com.br/doutrina/texto. asp?id=8702. Acesso em: 18 mar. 2019.

WESCHENFELDER, S. Caracterização de kefir tradicional quanto à composição físico-química, sensorial e atividade antiEscherichia coli. 2009. 72 f. Dissertação (Mestrado em Ciência e Tecnologia dos Alimentos) - Universidade Federal do Rio Grande do Sul, Porto Alegre, 2009.

WESCHENFELDER, S. et al. Caracterização físico-química e sensorial de kefir tradicional e derivados. Arquivo Brasileiro de Medicina Veterinária e Zootecnia, v. 63, n. 2, p. 473-480, 2011. DOI: 10.1590/S010209352011000200027

WESCHENFELDER, S. et al. Antibacterial activity of different formulations of cheese and whey produced with kefir grains. Revista Ciência Agronômica, v. 49, n. 3, p. 443-449, 2018a. DOI: 10.5935/1806-6690.20180050.

WESCHENFELDER, S. et al. Kefir: composição e avaliação da atividade antagonista in loco frente a Staphylococcus aureus e Escherichia coli. Revista Ciência Agronômica, v. 49, n. 3, p. 450-457, 2018 b. DOI: $10.5935 / 1806-6690.20180051$.

WSZOLEK, M. et al. Production of kefir, koumiss and other related products. Fermented milks. In: TAMIME, A. (ed.). Fermented Milks. Oxford: Blackwell Science, 2006. cap. 8, p. 174-216, 2006. DOI: 10.1002/9780470995501.ch8

ZENEBON, O.; PASCUET, N. S.; TIGLEA, P. (coord.). Métodos Físico-Químicos para Análise de Alimentos. 4. ed., 1. ed. digital. São Paulo: Instituto Adolfo Lutz, 2008.1020 p. 